\title{
Preventing the epidemic. Campaign to Control Cancer.
}

\author{
Aida Sivro (University of Manitoba) \\ News Reporter - HSI 2OIO-2OII
}

Next to cardiovascular disease, cancer has become one of the world's biggest killers. In 2007, almost 8 million people worldwide lost their lives prematurely due to cancer, and the number of cancer deaths is expected to increase almost $50 \%$ by $2030^{1}$. Based on the current incidence rates, an estimated $40 \%$ of Canadians will develop cancer during their lifetime ${ }^{2}$.

At the same time, the number of cancer deaths can be cut in half by applying knowledge we already possess. Worldwide, the most common types of cancer that kill men are lung, stomach, liver, colorectal and oesophagus, and for women, common types include: breast, lung, stomach, colorectal and cervical. One fifth of these cancers are caused by chronic infections, such as Human papillomavirus (HPV) that can lead to cervical cancer and hepatitis B (HBV) that has been linked to liver cancer. Additionally, tobacco use is the single largest preventable cause of cancer in the world.

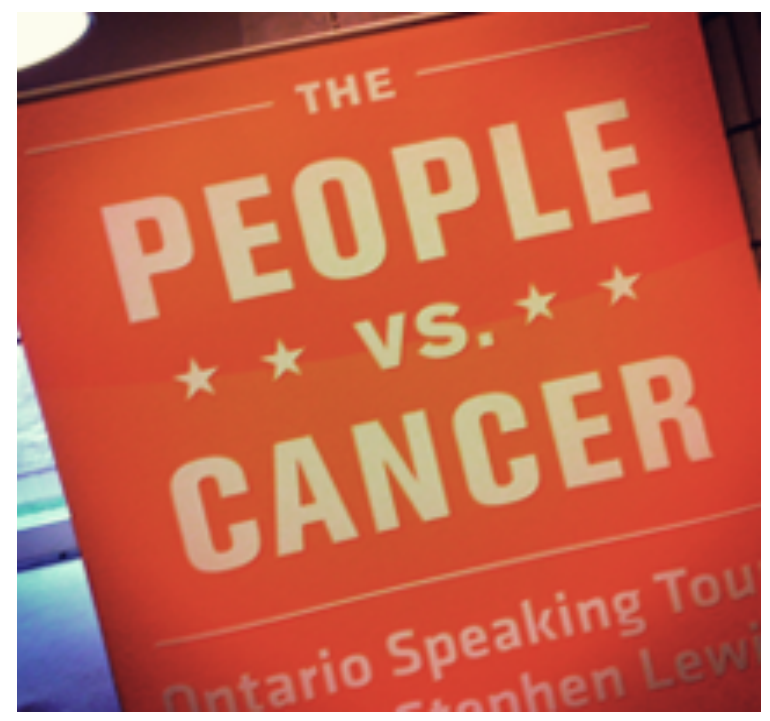

More than $30 \%$ of cancers can be prevented, mainly by avoiding tobacco use, having a healthy diet, being physically active and preventing cancer-causing infections. At the same time one third of cancers could be cured if detected and treated early. Even in late stage cancer, quality of life could be significantly improved if current knowledge about pain control and palliative care were applied more frequently.

Controlling cancer through knowledge change and action is something that people like Pat Kelly, CEO of the Campaign to Control Cancer (C2CC), have been advocating for years. Pat Kelly started her career in advocacy in 1987 when, as a young mother, she was diagnosed with breast cancer. Since then, Ms. Kelly has helped to establish networks of patient-support groups and has authored numerous publications, including six editions of the book What do we need to know about breast cancer.

The following are excerpts from an interview with Ms. Kelly regarding her work with C2CC and the "People vs. Cancer"- an Ontario Speaking Tour with Stephen Lewis. Organized by C2CC, the "People vs. Cancer" tour was brought to five Ontario universities. The tour also saw the launch of Community Conversations as a part of the Go Public initiative to raise public awareness, as well as provide a platform to share experiences and perspectives on cancer and cancer control.

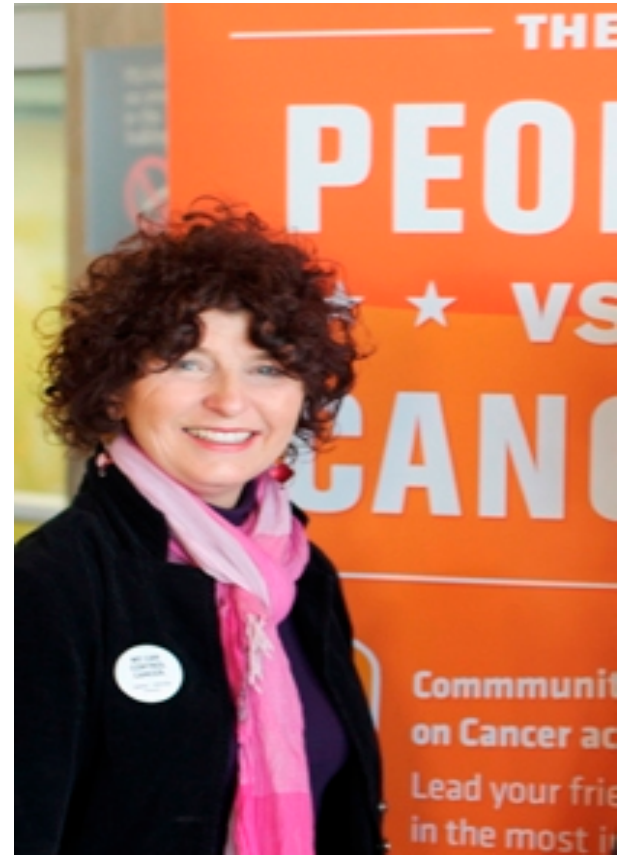

[AS] The "People vs. Cancer" - Ontario Speaking tour was joined by Stephen Lewis, the former Canadian ambassador to the UN and special UN envoy on HIV/AIDS in Africa. Why did you choose Mr. Lewis and what was his main message regarding cancer control?

[PK] Stephen Lewis has always been a champion of social justice. We have a lot of messages about cancer that go back almost a hundred years. In fact, they tend to be the same messages of investing in research and that our hope lies somehow in the future. In Canada and internationally, people are running for the cure or they are involved in public engagement efforts that focus on donations rather than on truly taking ownership of this idea that we can control cancer. It is not about bad luck, bad genes, and bad habits. We wanted a spokesperson who wasn't recognized as one of the traditional leaders within a cancer movement. In fact, we wanted somebody who is recognized as a leader in some other field to bring a different perspective to this idea. Stephen Lewis is an icon in the AIDS movement because his message is that people living with the disease have, in fact, the greatest capacity to influence and make change happen. And when you inspire others around a social justice issue, you build a momentum that is needed to make change happen. Canada has a national AIDS strategy in part because of people like Stephen Lewis, who said that it is not enough that people who live with this disease fight for themselves, the rest of us 
have to get involved. We wanted to have someone who had that capacity to galvanize, particularly young people, because that is where change will happen. The cancer movement has been dominated by cancer researchers and very conservative cancer charities, and the message about what taking control means is one that I do not think has been taken up by people of that generation. So the voice of Stephen Lewis, not a cancer activist but rather a social justice activist, and his message was: we can all take control of this; all of us have something that we can and will do.

[AS] Through the 'Community Conversations on Cancer', close to 1,500 Canadians participated in the Go Public initiative to characterize public awareness, experiences and perspectives on cancer. From this, the 2010 Report on Community Conversations ${ }^{3}$ states that more than half of the Canadians that participated could not correctly estimate their risk for cancer and were not aware that around half of cancers are preventable.

$[\mathrm{PK}]$ It is always surprising to learn that the prevalence of cancer is still not well understood within the population, and many elected officials that we meet with are surprised when we tell them that almost half of cancers are preventable. A lot of messages around cancer have been related to fundraising. Part of the challenge is to convert people from being donors or fundraisers to people who take action on an issue. We have to stop positioning cancer as a war because it sort of suggests that it is a win-loose environment. No! These small steps really make a difference: five to seven fruits and vegetables a day for you, for your kids, for your family; get screened; don't smoke; raise the cost of cigarettes and you really have a dramatic impact on youth smoking. Don't ignore what is right in front of you on your plate. What are the choices you make in the grocery store, because those will contribute as much to you and your family's risk for cancer as whether or not you live within a hundred yards of a power line.

\section{[AS] How can ordinary people and students help in the fight against cancer?}

[PK] We want you to join the Campaign to Control Cancer. Not just because we have something we want to get from you, but we believe that there is something you can give to this: your passion, your awareness, your creativity, your name, and your online presence. All of that will certainly influence your behavior and other people's behavior, but being part of the collective is what ultimately builds the momentum you need for social change. And students are in that time in life when you are making life style choices about diet, exercise, smoking, stress in your life, what you choose for your career. Many opportunities in oncology going forward; an aging population of baby boomers means there is going to be a lot of cancer, which means there are going to be a lot of jobs related to that. Look at the personal choices that you make, in terms of a career choice, look seriously at the options in health, social and political sciences and public policy, and join our campaign. Don't underestimate the power of students to have an impact.

Cancer affects everyone, rich and poor, young and old, men and women all over the world, and inflicts enormous strain on families and societies. While knowledge about cancer treatment and prevention is continually growing, the number of new cancer cases is increasing globally. It is time to translate current knowledge into action in order to save lives and improve quality of life. Each one of us has an important role to play in achieving a common goal - to control cancer, because the next life we save could be our own.

For more information and to join Campaign to Control Cancer visit: www.controlcancer.ca

1 World Health Organization, http://www.who.int/cancer/media/en/GlobalActionCancerEnglfull.pdf

2 Cancer Statistics Canada 2010

32010 Report on Community Conversations. http://www.controlcancer.ca/storage/cc2010-toolkit/national-snapshot.pdf

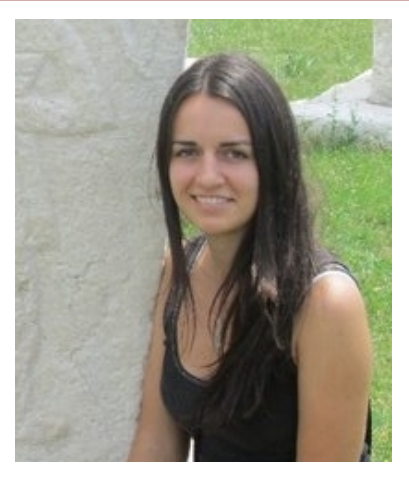

News Reporter Profile

Aida Sivro is currently persuing a PhD in Medical Microbiology at the University of Manitoba as part of the CIHR International Infectious Diseases and Global Health Training Program (IID \& GHTP). She is mainly interested in HIV immunology and the role host genetics plays in the susceptibility to HIV infection and rate of disease progression. 